\title{
A novel passenger recovery approach for the integrated airline recovery problem
}

\author{
Stephen J Maher \\ School of Mathematics and Statistics, University of New South Wales, Sydney NSW 2052, Australia. \\ Email: stephen.maher@unsw.edu.au, Phone: +61293856910
}

\begin{abstract}
Schedule disruptions require airlines to intervene through the process of recovery; this involves modifications to the planned schedule, aircraft routings, crew pairings and passenger itineraries. Passenger recovery is generally considered as the final stage in this process, and hence passengers experience unnecessarily large impacts resulting from flight delays and cancellations. Most recovery approaches considering passengers involve a separately defined module within the problem formulation. However, this approach may be overly complex for recovery in many aviation and general transportation applications. This paper presents a unique description of the cancellation variables that models passenger recovery by prescribing the alternative travel arrangements for passengers in the event of flight cancellations. The results will demonstrate that this simple, but effective, passenger recovery approach significantly reduces the operational costs of the airline and increases passenger flow through the network. The integrated airline recovery problem with passenger reallocation is solved using column-and-row generation to achieve high quality solutions in short runtimes. An analysis of the column-and-row generation solution approach is performed, identifying a number of enhancement techniques to further improve the solution runtimes.
\end{abstract}

Key words: airline recovery, passenger recovery, column generation, row generation.

\section{Introduction}

The efficient flow of passengers is critical to all transportation fields, especially when regular operations are affected by disruptions. The response to such a disruption is termed recovery, which involves strategies such as delaying and cancelling services in an effort to return to the originally planned state. Since transportation applications involve a number of interrelated resources, for example crew and aircraft in the airline context, each of these must be considered in recovery. Passenger are also of high importance, but their direct costs are not easily quantified. Consequently, passengers are not usually considered until the final stages of recovery processes. Hence, it is common for passengers to be significantly affected by 
any disruption in transportation applications.

The complete airline recovery process is a very large and complex problem that is commonly broken into a number of sequential stages. In practice, the sequential stages that form the airline recovery process are, the schedule, aircraft, crew and passenger recovery problems; however, it is common for the schedule and aircraft recovery to be performed simultaneously. Solving the airline recovery problem as series of discrete but related stages greatly reduces the problem complexity. These four stages are fundamental in the airline recovery process and provide the major focus areas of research. A very good recent review of the current airline recovery literature can be found in Clausen et al. [13].

The sequential solution approach for airline recovery involves solving each stage in isolation using the results from previous stages as input. Fixing the solution at each stage has the unfortunate effect of providing less flexibility to the problems solved later in this process. Consequently, it is common for the solutions to later stages to be suboptimal or even infeasible. The integration of multiple recovery stages is proposed as a method to address the possible suboptimality and infeasibility. The focus of this paper is an airline recovery problem that integrates schedule, aircraft, crew and passenger recovery. A major contribution of this paper is the modelling approach employed to consider passengers in the recovery process.

Airline planning and recovery processes and the flow of passengers is greatly affected by the network structure that is employed. There are two common types of airline networks, which are labelled as huband-spoke and point-to-point networks. The hub-and-spoke network identifies a number of key major airports (hubs) with a large proportion of all flights scheduled between these and outlying airports (spokes) and very few flights occurring between the spokes. This network is characterised by passenger itineraries, a set of flights booked to travel between an origin and destination, generally involving a transit through at least one hub. Alternatively, the point-to-point network is characterised by direct flights between most airports. As such, it is not common for passenger itineraries to involve more than one flight. The difference between these network types greatly affects the treatment of passengers during recovery. The attention towards point-to-point carriers in this paper is in response to the growing number of lowcost carriers providing this network structure and its relationship with other transportation applications, such as railway operations.

\subsection{Airline recovery}

The practicality of the sequential airline recovery process has driven the attention of researchers to review and develop individual recovery stages. Examples of the aircraft recovery problem, including schedule recovery decisions, are presented by Jarrah et al. [17], Cao and Kanafani [11,12], Bard et al. [6], Eggenberg et al. [15] and Rosenberger et al. [23]. The crew recovery problem has not received as much research attention compared to the aircraft problem, but many advancements have been made. Key examples of the crew recovery problem are presented by Wei et al. [28], Stojković et al. [26], Lettovsky et 
al. [19] and Abdelghaney et al. [2]. While the developments in each of the recovery stages have improved the overall recovery process, the issues of suboptimality and infeasibility still remain. These issues are only adequately addressed through the development of an airline recovery problem that integrates two or more of the sequential stages.

The development of the integrated airline recovery problem has been limited due to the problem complexity and difficulty in achieving fast solution runtimes. However, improvement in solution approaches and computing capabilities has prompted a recent surge of interest in this problem. An early proposal for solving the integrated recovery problem is provided by Lettovsky [18], which is based upon a Benders' decomposition framework. Lettovsky [18] presents a model that integrates the complete airline recovery problem; however, only the crew recovery problem has been implemented [19]. The modelling approach for the complete integrated recovery problem using Benders' decomposition is explored further by Petersen et al. [22]. In [22] a set of experiments for this approach are presented that achieve optimality within 30 minutes. An alternative, novel approach for the integrated recovery problem is presented by Abdelghany et al. [3] as an extension to the crew recovery problem of Abdelghany et al. [2]. This approach integrates aircraft, pilots and flight attendants with fast solution runtimes achieved by partitioning the schedule into sets of resource independent flights. While this partitioning process improves solution runtimes, a trade-off with the solution quality is observed through the overestimation of the optimal recovery costs. Finally, Maher [20] presents an airline recovery problem, integrating schedule, aircraft and crew. The focus of [20] is to improve the solution runtimes while maintaining a high solution quality using an exact solution approach. This is achieved with the application of a column-and-row generation framework that is presented in [20].

\subsection{Passenger recovery}

The vast majority of literature related to passenger recovery focuses on airlines operating a hub-andspoke network. As such, the passenger recovery process must reconstruct all disrupted itineraries, which may consist of multiple flight legs. This is a very complex and difficult task, since the number of possible itineraries is potentially much larger that the number of flights in the network. Additionally, this approach may be overly complex for airlines operating on a point-to-point network, where itineraries most commonly contain a single flight. The work presented in this paper introduces an alternative passenger recovery approach with a specific focus on airlines operating point-to-point networks. The modelling approach developed in this paper is a contribution to the airline passenger recovery literature and can be employed for alternative transportation applications, such as railway operations recovery.

One of the first models dedicated to the recovery of passenger itineraries is presented by Bratu and Barnhart [10]. In [10], two different optimisation models are described, analysing the trade-off between operating and passenger recovery costs, while also considering aircraft rerouting and the use of reserve crew. McCarty [21] presents an alternative passenger recovery approach that fits within the sequential 
recovery framework. The approach by [21] attempts to identify recovered itineraries for passengers in the event of a delay on one or many flights. The integration of the passenger and aircraft recovery problems is presented by Jafari and Zegordi [16]. The recovery problem in [16] uses the modelling approach of Abdelghany et al. [2] and Abdelghany et al. [3], where the recovery horizon is partitioned into sets of resource independent flights. The integrated aircraft and passenger recovery problem was also the focus of the 2009 ROADEF Challenge [1] which resulted in the development of many exact and heuristic solution approaches. The winning solution for this challenge is given by Bisaillon et al. [9], implementing a large neighbourhood search heuristic which identifies high quality solutions in short runtimes. Finally, the integrated airline recovery approaches of Lettovsky [18] and Petersen et al. [22] both consider the recovery of passenger itineraries as a dedicated Benders' decomposition subproblem.

The alternative passenger recovery approach presented in this paper attempts to directly provide passengers with alternative travel arrangements following flight cancellations. This involves redistributing passengers from cancelled flights to alternative operating flights, which may have been delayed, to ensure passengers arrive at their desired destination. This is achieved by introducing variables that describe both flight cancellations and the optimal redistribution of passengers. Such passenger reallocation methods are applicable to many transportation applications where passengers book single segment journeys, which is observed with low-cost airlines and railway operations. To the best of the authors knowledge, the modelling of the cancellation variables to describe passenger reallocation options has not been previously considered. A contribution of this paper is the development of a simple approach that efficiently recovers passengers while significantly reducing the expected recovery costs.

\subsection{Solution approaches}

Solution runtimes are a critical consideration of airline recovery problems, with reductions achieved through various approximation and decomposition approaches. Such approaches include the selection of specific recovery policies $[17,26,28]$, approximating flight arrival and departure times $[6,15]$ and the selection of affected aircraft [23] or crew $[2,19]$. In addition, decomposition techniques, such as column generation [15, 24-26] and Benders' decomposition [18,22], have also been employed to improve the solution runtimes. Finally, a heuristic approach is employed to solve the integrated airline recovery problem presented by Bisaillon et al. [9]. While these approaches successfully reduce the runtimes of the recovery problems, the heuristic approaches and techniques such as Benders' decomposition do not guarantee integer optimality.

An investigation into an exact solution approach to solve the integrated airline recovery problem is presented by Maher [20]. In [20], a general framework for column-and-row generation is developed and evaluated using the integrated airline recovery problem as an example. This framework is a direct alternative to Benders' decomposition, providing a guarantee of near integer optimal solutions. In addition, column-and-row generation is presented as a solution approach that reduces runtimes without 
the need to restrict the set of included crew and aircraft. While the general column-and-row generation framework presented by Maher [20] describes an approach to solve problems with multiple secondary variables and linking constraints, the application in [20] does not adequately evaluate such a framework. Hence, the implementation of column-and-row generation to a more general problem is not discussed.

In this paper, the consideration of passengers in the integrated airline recovery problem describes a problem with two sets of secondary variables and linking constraints. As such, the implementation of column-and-row generation discussed in this paper provides a more in-depth analysis of the framework presented in [20]. The discussion regarding the implementation and application of the framework from $[20]$ to a more general problem is a contribution of this paper.

\subsection{Outline of this paper}

The mathematical model for the integrated recovery problem with passenger reallocation (IRP-PR) is given in Section 2 with a description of the modelling approaches used for the included recovery policies. The column-and-row generation framework presented in Maher [20] is employed to solve the IRP-PR, with a description of its implementation provided in Section 3. This description will include a review of column generation and the integration with row generation in Sections 3.1 and 3.2 respectively. Section 4 will report the results from experiments, demonstrating the cost savings and improvement in passenger flow that can be achieved by this passenger recovery approach. Finally, a summary of the findings will be provided in Section 5 .

\section{Integrated Recovery Problem with Passenger Reallocation}

The integrated airline recovery problem with passenger reallocation (IRP-PR) integrates the schedule, aircraft and crew recovery problems with consideration to passenger flows through the recovered network. The integration of aircraft and crew recovery ensures that the optimal solution to both problems is found with consistent flight delay and cancellation decisions. The passenger considerations in the IRP-PR involves explicitly modelling the possible flight options that passengers can be rebooked onto in the event of a flight cancellation.

Passenger reallocation is a key aspect of the IRP-PR and the novel modelling approach is a major contribution of this paper. Following a flight cancellation passengers are either i) rebooked onto another flight operated by the airline, ii) rebooked onto another flight operated by a different airline, or iii) provided with a refund, including some compensation, and must rebook their own flight. Traditional airline recovery approaches ignore passengers until the end of the recovery process, which is equivalent to only modelling case iii). Consequently, schedule recovery decisions, such as delaying and cancelling flights, may be suboptimal for passenger flow. This paper presents an effective and efficient modelling approach for case i) in the integrated problem. The modelling approach employed involves reallocating 
passengers to alternative operating flights and calculating the flight cancellation costs to include this reallocation process.

The IRP-PR introduces three types of decision variables, described as the aircraft, crew and cancellation variables. The aircraft and crew variables detail the movement of these resources through the flight network. The cancellation variables describe the cancellation of a flight and the alternative flights that the passengers are rebooked onto. The specific details regarding the modelling of the cancellation variables is given in Section 2.3. The three variable types are linked in the IRP-PR by the flight delay and cancellation decisions and specific flights allocated to each aircraft and crew. An important aspect of the IRP-PR is the use of all aircraft and crew resources in the recovery problem, allowing for the optimal allocation of the available resources.

\section{$2.1 \quad$ Network structure}

The IRP-PR is solved for a single day flight schedule, attempting to completely recover from a disruption before to the end of the day. The airline recovery process is executed immediately following a schedule disruption, which is simply defined as anything preventing at least one flight from departing on time. As such, the flight schedule that is used in the IRP-PR, called the recovery schedule $N^{D}$, contains only a subset of all flights from the original flight schedule $N$. The construction of the recovery schedule $N^{D}$ uses a recovery window, defining the time period during which recovery actions can be performed. The recovery window commences immediately after the disruption and concludes at an arbitrary time that specifies when operations must return to plan. So the set $N^{D}$ is defined to contain all the flights in $N$ that depart after the disruption occurs but prior to the end of the recovery window.

In the IRP-PR, recovery actions are only permitted on flights contained in the set $N^{D}$, implying that the flights in $N \backslash N^{D}$ must depart as scheduled. To ensure that the flights contained in $N \backslash N^{D}$ are assigned crew and aircraft in the recovered solution, the concepts of carry-in and carry-out activities are introduced. The carry-in and carry-out activities define the expected position of each crew and aircraft at the start and end of the recovery period. These positions, generally stated as flights in the schedule, are identified from the sequences of flights assigned to each crew and aircraft in the planning solution. The carry-in activities for crew and aircraft, contained in $N_{i n}^{K}$ and $N_{i n}^{R}$, are the flights in each sequence that are performed immediately prior to the disruption. These activities may also include origination airports if the crew or aircraft have not commenced the days work prior to the disruption. A carry-out activity for crew and aircraft, contained in $N_{\text {out }}^{K}$ and $N_{\text {out }}^{R}$, are the flights in each sequence that are performed immediately after the end of the recovery window. Similar to the carry-in activities, a carry-out activity may be defined as a termination airport if the crew or aircraft is expected to finish working within the recovery window. The set of all origination and termination airports $t$ are contained in $T^{K}$ and $T^{R}$ for crew and aircraft respectively.

Flight delays are implemented in the IRP-PR using the technique of flight copies. The implementation 
of flight copies employed in this paper is identical to that presented in Maher [20]. This technique models flight delays by creating multiple copies of each flight within the schedule, each with a later departure time. The set of flight copies $v$ defined for each flight $j \in N$ are contained in the set $U_{j}$. Since the flights $j \in N \backslash N^{D}$ must depart as originally scheduled, the set of copies only includes the copy representing the original departure time, i.e. $U_{j}=\{0\}$. For the disruptable set of flights, $j \in N^{D}$, the set of allowable copies contains additional copies $v_{i}, i=1, \ldots, n$ to represent flight delays, i.e. $U_{j}=\left\{0, v_{1}, \ldots, v_{n}\right\}$. For convenience, the notation $j_{v}$ is used to define the flight-copy pair representing the delayed departure of flight $j$ given by copy $v$. Using this definition, all flight-copy pairs for the original and recovery schedule are contained in $\hat{N}$ and $\hat{N}^{D}$ respectively, which includes all flights and origination and termination locations.

Every flight in the schedule is defined by an origin and destination with respective departure and arrival times. Implementing delays using flight copies provides a number of alternative departure and arrival times for each flight $j$ given by the set $U_{j}$. The nodes of the connection network are represented by each flight-copy pair contained in $\hat{N}^{D}$ and the arcs are supplied by the connections between each of the nodes. A feasible connection between two flight-copy pairs $\left(i_{u}, j_{v}\right)$ exists if i) the origin of flight $j$ is the same as the destination of flight $i$, and ii) the difference between the departure of flight-copy $j_{v}$ and the arrival of flight-copy $i_{u}$ is greater than a minimum ground time. For aircraft the ground time is called the turn time and for crew it is the sit time. Since there are different conditions that dictate the feasible connections for crew and aircraft, the connection networks for the disruptable schedule are defined as $C^{K}$ and $C^{R}$ respectively.

The minimum turn time for aircraft is shorter than the minimum sit time for crew, as such all permissible connections for crew are also permissible for aircraft. The set of connections with a ground time between the minimum turn and sit times are defined as short connections, given by $E=C^{R} \backslash C^{K}$. While the ground time for these connections is less than the minimum sit time, crew can be assigned to connection $\left(i_{u}, j_{v}\right) \in E$ if an aircraft is also assigned to this connection. This is a critical aspect of the integration between the aircraft and crew recovery problems which greatly improves upon the solution quality achieved by the comparative sequential recovery process.

\section{$2.2 \quad$ Flight string variables}

A flight string formulation introduced by Barnhart et al. [7] is used to model the crew and aircraft variables in the IRP-PR. Flight strings constructed for all crew $K$ and aircraft $R$ form a sequence of connected flights from a carry-in to a carry-out activity. All flight strings representing the flow of each crew $k$ and aircraft $r$ through the connection network are contained in the sets $P^{k}$ and $P^{r}$ respectively. The variables $x_{p}^{k}$ equal 1 if flight string $p$ is operated by crew $k$ and the variables $y_{p}^{r}$ are similarly for aircraft. The cost of a flight string for crew $k, c_{p}^{k}$, represents the additional work performed, measured in terms of work hours, as a result of a disruption and the subsequent recovery actions. Similarly, the 
cost of a flight string for aircraft $r, c_{p}^{r}$, describes the cost of delaying any flights that are contained in string $p$.

The parameters $a_{j p}^{k}$ and $a_{j p}^{r}$ equal 1 if flight $j$ is included on flight string $p$ for crew $k$ and aircraft $r$ respectively. To indicate the use of short connection $\left(i_{u}, j_{v}\right)$ on flight string $p$, the parameters $b_{i_{u} j_{v} p}^{k}$ and $b_{i_{u} j_{v} p}^{r}$ equal 1 for crew $k$ and aircraft $r$ respectively. Also, $a_{j p}^{k v}$ and $a_{j p}^{r v}$ equal 1 to indicate that flight $j$ on string $p$ is delayed by a length of time given by copy $v$ for crew $k$ and aircraft $r$ respectively. In addition to defining a sequence of flights performed throughout the day, an aircraft flight string also specifies the termination airport with the parameters $o_{t p}^{r}$, that equal 1 if the flight string $p$ for aircraft $r$ terminates at overnight airport $t$.

An important aspect of crew flight strings is the legality with respect to various work rules. There are numerous rules that dictate the construction of legal crew flight strings, related to duties, pairings and schedules. A review of the crew scheduling process, including a detailed overview of the relevant crew work rules is given in Barnhart et al. [8]. Since the IRP-PR is solved for a single day schedule, only the crew duty rules are considered in this paper.

The crew duty rules modelled in the IRP-PR are station compatibility requirements and an upper bound on the number of flying and working hours. First, the station compatibility requirements state the airports where crew may start and finish their work day. In particular, crew are employed from one of many crew bases located throughout the network. To ensure station compatibility, it is ideal for crew to originate and terminate their duties at the same base. However, this is not always achieved as a result of the flight schedule design and crew may terminate at a permissible overnight airport. Second, an upper bound on the number of working and flying hours are set to manage the effects of fatigue on crew. In the IRP-PR the maximum number of flying hours and working hours are set to 8 and 13 respectively, which have been adopted from Barnhart et al. [8]. Finally, disruptions can occur at any point during the day, as such it is important to account for the work history performed by each crew prior to the disruption. This is achieved by constructing personalised duties for each crew that satisfy the working rules with respect to any work performed before and after the disruption.

\subsection{Cancellation variables - passenger recovery by reallocation}

Passenger reallocation is a process whereby passengers booked on cancelled flights are rebooked onto alternative flights. These alternative flights are termed reallocation options and may still be operating as scheduled or delayed. This process accounts for the number of seats remaining on the reallocation options and the total length of delay to be experienced by the reallocated passengers. Considering passenger reallocation allows the optimisation process to make cancellation and delay decisions while attempting to improve passenger flow. In the traditional, sequential airline recovery process, passenger reallocation is performed using a greedy approach that will be shown to be suboptimal in Section 4.2.1.

The conventional approach used to model flight cancellations is with a single variable per flight that 
equal 1 to indicate a cancellation and 0 otherwise. In the IRP-PR, the variable $z_{j p}$ is defined to indicate the cancellation of flight $j$ but also the reallocation of passengers to alternative flights as given by scheme $p$. Since there are multiple reallocation options following the cancellation of each flight $j$, the set $P^{j}$ is defined to contain each passenger reallocation scheme $p$. The cost of a reallocation scheme $p$ for flight $j$ is $d_{j p}$, which attempts to quantitatively describe the reduced passenger satisfaction resulting from the delay experienced by the reallocated passengers and not providing alternative travel arrangements to the remaining.

To conveniently describe the passenger reallocation schemes in the IRP-PR, the sets $N_{i}^{\text {post }}$ and $N^{j}$ are defined to contain the alternative flights for disrupted passengers. The alternative flights for passengers following the cancellation of flight $i$ are identified by having the same origin-destination (O-D) pair as $i$ and depart within a time window commencing from the departure time of $i$. Using this definition, the set $N_{i}^{\text {post }}$ contains all flights $j$ that are travel alternatives for passengers booked on flight $i$. Conversely, the set $N^{j}$ contains all flights $i$ for which flight $j$ is a travel alternative.

Each of the reallocation schemes $p$ for the cancellation variables can be likened to the solution of a knapsack problem. For example, following the cancellation of flight $i$ the size of the knapsack is given by the number passenger booked on that flight, $\operatorname{Pax}(i)$, and the items to enter the knapsack are the available seats $r_{j}=$ Maxcap $-\operatorname{Pax}(j)$ on alternative flights $j \in N_{i}^{\text {post }}$. In the model constraints, the number of passengers reallocated to the flight-copy pair $j_{v}$ following the cancellation of flight $i$ on reallocation scheme $p$ is given by $h_{i j p}^{v}$.

\section{$2.4 \quad$ Recovery policies}

The recovery policies implemented in the IRP-PR are flight delays and cancellations, crew deadheading, the use of reserve crew and the generation of new crew duties and aircraft routes. This set of recovery policies is representative of the actions taken by airlines in practice. In Section 2.1, the concept of flight copies is presented as the approach used in the IRP-PR to model flight delays. The cost of flight delays attempts to model the effect of the disruption on passenger satisfaction. This is included in the objective function through the cost of aircraft flight strings, $c_{p}^{r}$. An unfortunate effect of performing flight delays is that airport arrival capacities, the number of permitted arrivals in a given time period, may be violated. This aspect related to recovery is considered by defining $L$ as the set of all airports and $W$ as the set of time windows that partition the complete recovery period. Thus, the number of aircraft arrivals at airport $l \in L$ within time window $w \in W$ is restricted to $A_{l}^{w}$.

As a result of flight cancellations the flow balance of the schedule is destroyed requiring a lower bound to be imposed on the number of aircraft that must terminate at each overnight base. This lower bound is set from the solution to the planning stage and is given by $\sum_{r \in R} \bar{o}_{t}^{r}$. The parameter $\bar{o}_{t}^{r}$ describes whether the planned flight string for aircraft $r$ terminates at airport $t$ within the recovery period.

The recovery of crew introduces two specific recovery policies, namely deadheading and the use of 
reserve crew. Within the crew deadheading recovery policy, there are two different approaches implemented for the IRP-PR, involving the transportation of crew as passengers to either i) return them to their crew base immediately following the start of a disruption, or ii) reposition them to continue the operation of disrupted duties. The first approach is called deadheading crew back to base, which is modelled by the variables $\nu_{k}$ that equal 1 to indicate crew $k$ is transported directly back to base immediately following the disruption. In the IRP-PR, deadheading crew back to their crew base is performed at a cost of $g^{D H B}$. The second approach permits crew to deadhead within a duty to another airport to continue the operation of other disrupted duties. This deadheading action is modelled in the IRP-PR by the variables $\kappa_{j}^{v+}$ that count the number of crew deadheading on flight copy $j_{v}$. The dummy variables $\kappa_{j}^{v-}$ are introduced to ensure that the number of crew deadheading on flight-copy $j_{v}$ is exactly one less than the number of crew assigned to that flight. The cost of deadheading one crew member on a single flight leg within a duty is given by $g^{D H D}$.

The use of reserve crew is a very important aspect of crew recovery, providing additional resources to operate the perturbed flight schedule. Reserve crew $k \in K^{r e s} \subset K$ are employed in the recovery process to avoid the regular crew exceeding the allowable working hours and to minimise the number of cancelled flights. The reserve crew are located at each crew base and can be assigned flight strings that originate from their base. The cost of a duty $p$ for reserve crew $k, c_{p}^{k}$, represents the cost of any work performed including a fixed penalty to minimise their use.

The mathematical model of the IRP-PR is given by,

$$
\begin{aligned}
& \min \sum_{k \in K} \sum_{p \in P^{k}} c_{p}^{k} x_{p}^{k}+\sum_{j \in N^{D}} \sum_{v \in U_{j}} g^{D H D} \kappa_{j}^{v+}+\sum_{k \in K} g^{D H B} \nu_{k}+\sum_{r \in R} \sum_{p \in P^{r}} c_{p}^{r} y_{p}^{r}+\sum_{j \in N^{D}} \sum_{p \in P^{j}} d_{j p} z_{j p}, \\
& \text { s.t. } \sum_{k \in K} \sum_{p \in P^{k}} a_{j p}^{k} x_{p}^{k}-\sum_{v \in U_{j}} \kappa_{j}^{v+}+\sum_{p \in P^{j}} z_{j p}=1 \quad \forall j \in N^{D} \text {, } \\
& \sum_{k \in K} \sum_{p \in P^{k}} a_{j p}^{k v} x_{p}^{k}-\kappa_{j}^{v+}+\kappa_{j}^{v-}=1 \quad \forall j \in N^{D}, \forall v \in U_{j}, \\
& \sum_{k \in K} \sum_{p \in P^{k}} a_{j p}^{k} x_{p}^{k}=1 \quad \forall j \in N_{o u t}^{K}, \\
& \sum_{r \in R} \sum_{p \in P^{r}} a_{j p}^{r} y_{p}^{r} \leq 1 \quad \forall j \in N^{D} \\
& \sum_{r \in R} \sum_{p \in P^{r}} a_{j p}^{r} y_{p}^{r}=1 \quad \forall j \in N_{o u t}^{R}, \\
& \sum_{r \in R} \sum_{p \in P^{r}} o_{t p}^{r} y_{p}^{r} \geq \sum_{r \in R} \bar{o}_{t}^{r} \quad \forall t \in T^{R} \\
& \sum_{r \in R} \sum_{p \in P^{r}} v_{l p}^{r w} y_{p}^{r} \leq A_{l}^{w} \quad \forall l \in L, \forall w \in W \\
& \sum_{k \in K} \sum_{p \in P^{k}} b_{i_{u} j_{v} p}^{k} x_{p}^{k}-\sum_{r \in R} \sum_{p \in P^{r}} b_{i_{u} j_{v} p}^{r} y_{p}^{r} \leq 0 \quad \forall\left(i_{u}, j_{v}\right) \in E^{D}, \\
& \sum_{k \in K} \sum_{p \in P^{k}} a_{j p}^{k v} x_{p}^{k}-\kappa_{j}^{v+}-\sum_{r \in R} \sum_{p \in P^{r}} a_{j p}^{r v} y_{p}^{r}=0 \quad \forall j \in N^{D}, \forall v \in U_{j}
\end{aligned}
$$




$$
\begin{gathered}
\left\{\sum_{k \in K} \sum_{p \in P^{k}} a_{j p}^{k v} x_{p}^{k}-\kappa_{j}^{v+}\right\} r_{j}-\sum_{i \in N^{j}} \sum_{p \in P^{i}} h_{i j p}^{v} z_{i p} \geq 0 \\
\sum_{p \in P^{k}} x_{p}^{k}+\nu_{k}=1 \quad \forall j \in N^{D}, \forall v \in U_{j}, \\
\sum_{p \in P^{k}} x_{p}^{k} \leq 1 \quad \forall k \in K^{r e s}, \\
\sum_{p \in P^{r}} y_{p}^{r}=1 \quad \forall r \in R, \\
x_{p}^{k} \in\{0,1\}, \forall k \in K, \forall p \in P^{k}, \quad y_{p}^{r} \in\{0,1\}, \forall r \in R, \forall p \in P^{r}, \\
z_{j p} \in\{0,1\}, \forall j \in N^{D}, p \in P^{j}, \quad \nu_{k} \in\{0,1\}, \forall k \in K, \\
\kappa_{j}^{v+} \geq 0, \kappa_{j}^{v-} \geq 0 \quad \forall j \in N^{D}, \forall v \in U_{j} .
\end{gathered}
$$

The objective of the IRP-PR is to minimise the additional operational cost associated with the recovery of crew and aircraft and the reallocation of disrupted passengers. This involves minimising the costs related to crew deadheading, flight delays and cancellations and any work performed by crew in addition to that originally planned. Another common objective for recovery problems is to minimise the number of changes between the recovered and the planned solution [27]. This is implemented in the IRP-PR by subtracting a bonus from the cost of crew pairings and aircraft routes for each connection $(i, j)$ used in the recovery solution that is also used in the planning solution.

Crew recovery in the integrated problem is described by constraints (2)-(4) and (12)-(13). The flight coverage constraints (2) ensure that every flight is either assigned to a crew group or cancelled. The term $\sum_{v \in U_{j}} \kappa_{j}^{v+}$ in (2) is the total number of crew deadheading on flight $j$. The inclusion of constraint (3) ensures that at most one crew group is operating flight-copy $j_{v}$, i.e. all other crew assigned to $j_{v}$ are deadheading, which is required to implement crew deadheading within a duty. Cancellation is not permitted for the carry-out activities, so each must be assigned a crew group, as defined by constraints (4). Constraints (12) ensure that all originally planned crew, $K \backslash K^{\text {res }}$, are either assigned to a recovered flight string or deadheaded back to their crew base. By contrast, reserve crew are only required in exceptional circumstances which is indicated by the inequality in constraints (13).

The structure of aircraft recovery in the IRP-PR, given by (5)-(8) and (14), is similar to the crew recovery detailed above. Flight coverage is described by constraints (5) and the coverage of carry-out activities is enforced by constraint (6). The difference between the flight coverage constraints of crew (2) and aircraft (5) is that the cancellation variables are not required in the latter. This is due to the consistency of flight cancellations being enforced by other constraints in the model. An additional feature for the aircraft recovery problem is constraint (7), which specifies the minimum number of aircraft flight strings that must terminate at each overnight airport within the recovery period. Also, the number of arrivals permitted at each airport within a given time window is restricted by constraints (8). Finally, all aircraft that are assigned a routing in the planned solution must be assigned a recovered flight string, 
which is captured by constraint (14).

The integration of crew and aircraft in the IRP-PR is described by the use of short connections and consistency of flight delays. As stated in Section 2.1, it is permissible for crew to use a short connection if that same connection is used by aircraft, which is described by constraints (9). Additionally, in the integrated problem it is important to ensure that the amount of delay on each flight is consistent between crew and aircraft, which is enforced by constraints (10).

As a variation on the conventional modelling approach, the cancellation of flight $i$ is indicated by $\sum_{p \in P^{i}} z_{i p}=1$ in (2). The contribution of this modelling approach is that if $\sum_{p \in P^{i}} z_{i p}=1$, flight $i$ is cancelled and the booked passengers are reallocated to alternative flights, which are contained in $N_{i}^{\text {post }}$, as described by constraint (11). This set of constraints is in the form of big-M constraints, only permitting the reallocation of passengers to flight $j$ if crew are also operating that flight. The total number of passengers that can be reallocated to flight $j$ is given by $r_{j}=\operatorname{Maxcap}-\operatorname{Pax}(j)$. To the best of the authors knowledge this modelling approach for passenger recovery has not previously been considered.

\section{Solution Methods}

Airline operations are very dynamic with the current state of the system changing almost every minute. In any transportation application, it is necessary for recovery problems to achieve high quality solutions in very short runtimes. A general framework for column-and-row generation is developed and applied in Maher [20], demonstrating reduced runtimes for the integrated airline recovery problem (IRP). Unfortunately, the IRP presented in [20] only applies a special case of the developed column-and-row generation framework, namely the problem is formulated with only one set of secondary variables and linking constraints. Hence, solving the IRP does not adequately assess the potential of the solution approach. The extension presented by the IRP-PR introduces additional variables and constraints related to the cancellation variables that further increases the complexity of the recovery problem. The inclusion of this set of variables and constraints formulates a problem to which the general column-and-row generation framework of [20] can be applied. The application of column-and-row generation described in this section and the evaluation presented in Section 4 is a contribution of this paper.

The column-and-row generation solution approach will be presented in relation to the key components of column generation and row generation. Section 3.1 will discuss the application of column generation to solve the IRP-PR. This discussion is provided assuming that the IRP-PR is solved by column generation alone. A focus of Section 3.1 is the column generation subproblem for the cancellation variables, given in Section 3.1.2. Finally, Section 3.2 will describe the application of the row generation procedure from the column-and-row generation framework developed in [20] to the IRP-PR. 


\subsection{Column generation}

Column generation relies on the understanding that the vast majority of variables are non-basic in the optimal solution. Thus, a restricted master problem (RMP) is initially formulated to contain only a subset of all variables from the IRP-PR. This initial subset of variables is denoted by $\bar{P}^{k} \subseteq P^{k}, \bar{P}^{r} \subseteq P^{r}$ and $\bar{P}^{i} \subseteq P^{i}$ for the crew, aircraft and cancellation variables respectively. The RMP is solved to identify an upper bound on the original problem, which is progressively improved with the addition of variables. The following sections will detail the column generation subproblems solved to identify variables that are expected to improve the upper bound given by the current formulation of the RMP.

To aid the discussion in this section the notation for the dual formulation of RMP will be provided. The dual variables for the flight coverage constraints (2) and (4), for crew; and (5) and (6), for aircraft; are defined as $\boldsymbol{\alpha}^{K}=\left\{\alpha_{j}^{K}, \forall j \in N^{D} \cup N_{\text {out }}^{K}\right\}$ and $\boldsymbol{\alpha}^{R}=\left\{\alpha_{j}^{R}, \forall j \in N^{D} \cup N_{\text {out }}^{R}\right\}$ respectively. The dual variables for the surplus crew count constraints (3) are defined by $\boldsymbol{\eta}=\left\{\eta_{j}^{u}, \forall j \in N^{D}, \forall u \in U_{j}\right\}$. The dual variables $\boldsymbol{\epsilon}=\left\{\epsilon_{t}, \forall t \in T\right\}$ are defined for the aircraft end-of-day location constraints (7). The dual variables for the aircraft movement constraints (8) are given by $\boldsymbol{\beta}=\left\{\beta_{l}^{w}, \forall l \in L, \forall w \in W\right\}$. The dual variables for the short connection constraints (9), delay consistency constraints (10) and the passenger reallocation constraints (11) are given by $\boldsymbol{\rho}=\left\{\rho_{i j}, \forall(i, j) \in E^{D}\right\}, \gamma=\left\{\gamma_{j}^{u}, \forall j \in N^{D}, \forall u \in U_{j}\right\}$ and $\boldsymbol{\lambda}=\left\{\lambda_{j}^{v}, \forall j \in N^{D}, \forall u \in U_{j}\right\}$ respectively. Finally, the dual variables $\boldsymbol{\delta}^{K}=\left\{\delta^{k}, \forall k \in K\right\}$ and $\boldsymbol{\delta}^{R}=\left\{\delta^{r}, \forall r \in R\right\}$ are defined for the crew and aircraft assignment constraints, (12)-(13) and (14), respectively. The column generation subproblems for the crew and aircraft variables are formulated as shortest path problems, given in Section 3.1.1. Finally, the subproblem for the cancellation variables can be formulated as a bounded knapsack problem, which is described in Section 3.1.2.

\subsubsection{Shortest Path Subproblem}

The column generation subproblems for the crew duty and aircraft routing variables are described by;

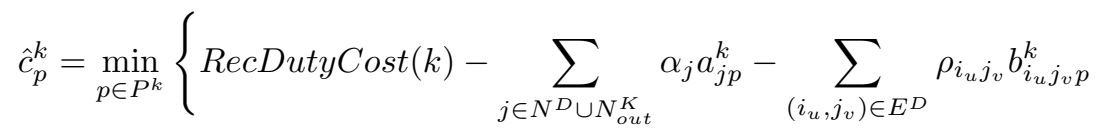

$$
\begin{aligned}
& \left.-\sum_{j \in N^{D}} \sum_{v \in U_{j}}\left\{\eta_{j}^{v}+\gamma_{j}^{v}+r_{j} \lambda_{j}^{v}\right\} a_{j p}^{k v}-\delta^{k}\right\}, \quad \forall k \in K, \\
& \hat{c}_{p}^{r}=\min _{p \in P^{r}}\left\{\sum_{j \in N^{D}} \sum_{v \in U_{j}} c_{j p}^{r v} a_{j p}^{r v}-\sum_{j \in N^{D \cup N_{\text {out }}^{K}}} \alpha_{j} a_{j p}^{r}-\sum_{t \in T} \epsilon_{t} o_{t p}^{r}-\sum_{l \in L} \sum_{w \in W} \beta_{l}^{w} v_{l p}^{r w}\right. \\
& \left.+\sum_{\left(i_{u}, j_{v}\right) \in E^{D}} \rho_{i_{u} j_{v}} b_{i_{u} j_{v} p}^{r}+\sum_{j \in N^{D}} \sum_{v \in U_{j}} \gamma_{j}^{v} a_{j p}^{r v}-\delta^{r}\right\}, \quad \forall r \in R .
\end{aligned}
$$

In problems (18) and (19), the minimisation problem is defined over the sets $P^{k}$ and $P^{r}$ which contain all possible flight strings for crew $k$ and aircraft $r$ respectively. While this description indicates that additional variables can be identified from the sets $\bar{P}^{k}$ and $\bar{P}^{r}$, these sets represent variables included 
in the master problem, hence their reduced costs are at least zero. Therefore, all negative reduced cost variables identified by solving (18) and (19) will be contained in $P^{k} \backslash \bar{P}^{k}$ and $P^{r} \backslash \bar{P}^{r}$.

The set of all aircraft flight strings are contained in $P^{r}, \forall r \in R$ and are described as a sequence of connected flight between carry-in and carry-out activities. The sets $P^{r}, \forall r \in R$ can be defined as the feasible region of a network flow problem. The connection network used in the shortest path problem for aircraft is constructed with a single source node and multiple sink nodes, described by $\hat{N}^{D}$ representing the nodes and $C^{R}$ representing the arcs. In addition, maintenance is the only requirement that must be enforced in the construction of feasible flight strings. For each aircraft that requires maintenance at the end of the day, all flight strings are constructed to terminate at a maintenance station. Therefore, only the single resource of cost is propagated through the network. The cost of an aircraft flight string is given by $c_{p}^{r}=\sum_{j \in N^{D}} \sum_{v \in U_{j}} c_{j p}^{r v} a_{j p}^{r v}$, which is the simple sum of the delay costs for each flight operated by aircraft $r$. There are various standard solution algorithms available to solve this problem, many of which are described in Ahuja et al. [5].

While a feasible flight string for crew is also a sequence of connected flights, there are specific rules governing the construction. The crew duty rules modelled in the IRP-PR are described in Section 2.2 and are enforced in the column generation subproblems. Each of the flight strings contained in $P^{k}$ respect the origination and termination location and the restriction on the maximum number of working and flying hours. As a consequence of the complex crew duty rules, the column generation subproblem for crew presents a more complex shortest path problem than the problem solved for aircraft.

The objective function for problem (18) represents the reduced cost for crew duty variables. The cost of a crew duty is a max function related to the flying hours, fly, the total working hours, work, a minimum number of guaranteed hours, minGuar, and the cost of the originally planned duty for crew $k$, OrigDutyCost $(k)$. This function is based off the crew duty cost presented by Barnhart et al. [8] and is given by,

$$
\operatorname{RecDutyCost}(k)=\max \left\{0, \max \left\{f l y, f_{d} \cdot \text { work, } \operatorname{minGuar}\right\}-\operatorname{OrigDutyCost}(k)\right\},
$$

where minGuar is set at 6 hours [8] and $f_{d}$ is a fraction which is airline specific and is set at $f_{d}=5 / 8[8]$ for the IRP-PR. The cost of a recovered crew duty given by equation (20) is the cost associated with any additional work performed as a result of a disruption and the respective recovery actions. In the case of reserve crew, the original duty cost is set to zero and a fixed penalty is added to RecDutyCost $(k)$.

A benefit of column generation is that all feasible flight strings contained in $P^{k}, \forall k \in K$ are not enumerated a priori but defined as the feasible region of a network flow problem. This conveniently allows for negative reduced cost paths to be identified by the solution to a shortest path problem. For the crew duty variables, the shortest path problem is solved on a connection network with one source node and multiple sink nodes, which is described by $\hat{N}^{D}$ representing the nodes and $C^{K} \cup E^{D}$ representing the arcs. Since there are a numerous rules that must be reviewed to construct feasible crew flight strings, a multi-label shortest path problem is used to track the resources of cost, flying and working hours. By 
tracking multiple resources through the connection network, an efficient frontier is constructed at each node by all labels that are propagated to there. The use of multiple labels in the shortest path problem allows the propagation paths that are suboptimal with respect to cost but have a resource consumption that lies on the efficient frontier. It is possible to adapt the algorithm used for aircraft to develop an efficient solution approach for the crew column generation subproblem.

General aircraft column generation subproblem. The column-and-row generation solution approach requires the solution to the shortest path problem for aircraft to construct an optimal dual solution. It is observed in problem (19) that all dual variables, except for $\boldsymbol{\delta}^{R}$, are aircraft independent. Therefore, by setting $\delta^{r}=\delta^{R}, \forall r \in R$, where $\delta^{R}=\max _{r \in R}\left\{\delta^{r}\right\}$ in (19), it is possible to formulate a single, aircraft-independent shortest path problem, which is labelled $\operatorname{PSP}^{R}$. The solution to the $\operatorname{PSP}^{R}$ is a lower bound on the minimum reduced cost flight string for all aircraft. This problem is implemented in the row generation procedure, which is described in Section 3.2.2.

\subsubsection{Cancellation variable subproblem}

The modelling of the cancellation variables is unique in regards to the airline context and the column generation implementation. While there are many application of column generation where the subproblem is defined as one of the many knapsack problem variants, there are few where the items to enter the knapsack are duplicated multiple times with slightly different properties. For the IRP-PR, the size of the knapsack is given by the passengers on the cancelled flight $i$ and the items are the seats on the alternative flights contained in $N_{i}^{\text {post }}$. Since flight delays are implemented using flight-copies, it is possible for many identical flights to be contained in $N_{i}^{\text {post }}$ that differ only by the departure time. The treatment of this unique formulation of the column generation subproblem will be discussed in this section.

Each cancellation variable defines a reallocation scheme providing alternative travel arrangements for passengers in the event of a flight cancellation. Ideally this modelling approach provides an alternative travel arrangement for all disrupted passengers, however this is not always possible. As such, passengers on cancelled flights are partitioned into two categories, stranded and reallocated passengers. To model the cost of a flight cancellation $d_{i p}$, a cost is assigned to each of these categories. Namely, the parameter $g^{C A N}$ is defined as the cost of leaving a single passenger stranded and $g_{i j}^{R A}$ as the cost of reallocating a single passenger to flight $j$ following the cancellation of flight $i$. Both the costs given by $g^{C A N}$ and $g_{i j}^{R A}$ include a quantitative measure of passenger dissatisfaction and loss of good will. The definition of $d_{i p}$ for the reallocation of passengers by scheme $p$ following the cancellation of flight $i$ is given by,

$$
d_{i p}=g^{C A N} \operatorname{Pax}(i)+\sum_{j \in N_{i}^{\text {post }}} \sum_{v \in U_{j}} h_{i j p}^{v}\left(g_{i j}^{R A}-g^{C A N}\right) .
$$

Equation (21) consists of a fixed cost which assumes all passengers are stranded and a variable cost that is dependent on the number of passengers rebooked onto alternative flights. 
The cost of a service cancellation given by equation (21) can be applied in many different contexts. For example, in public transport applications the fixed cost for cancelling a service, $g^{C A N}$, can be set to zero since all waiting passengers will travel on the next available service. However, due to overcrowding the variable cost, $g_{i j}^{R A}$, can be very high. Given the similarities in the cost structure, the proposed passenger recovery approach can also be applied.

Using the definitions of the dual variables given in Section 3.1, the reduced cost of a cancellation variable for flight $i$ is given by,

$$
\bar{d}_{i p}=g^{C A N} \operatorname{Pax}(i)-\alpha_{i}^{K}+\sum_{j \in N_{i}^{\text {post }}} \sum_{v \in U_{j}} h_{i j p}^{v}\left(\lambda_{j}^{v}+g_{i j}^{R A}-g^{C A N}\right)
$$

It is observed that the first two terms of equation (22) are not dependent on the reallocation of passengers to alternative flights. Therefore, the objective function for the cancellation variables column generation subproblem is defined only by the final term. The column generation subproblem for the cancellation variables (CV-PSP) to identify passenger reallocation schemes for flight $i$ is given by,

$$
\begin{aligned}
\tilde{d}_{i p}=\max & \sum_{j \in N_{i}^{\text {post }}} \sum_{v \in U_{j}} h_{i j p}^{v}\left(g^{C A N}-\lambda_{j}^{v}-g_{i j}^{R A}\right), \\
\text { s.t. } & \sum_{j \in N_{i}^{\text {post }}} \sum_{v \in U_{j}} h_{i j p}^{v} \leq \operatorname{Pax}(i), \\
& h_{i j p}^{v} \leq r_{j} w_{i j}^{v} \quad \forall j \in N_{i}^{\text {post }}, \forall v \in U_{j}, \\
& \sum_{v \in U_{j}} w_{i j}^{v}=1 \quad \forall j \in N_{i}^{\text {post }}, \\
& h_{i j p}^{v} \in \mathbb{Z}^{+} \quad \forall j \in N_{i}^{\text {post }}, \forall v \in U_{j}, \\
& w_{i j}^{u} \in\{0,1\} \quad \forall j \in N_{i}^{\text {post }}, \forall v \in U_{j} .
\end{aligned}
$$

The problem described by CV-PSP shares many characteristics with a bounded knapsack problem. The main difference between the CV-PSP and a bounded knapsack problem arises from the multiple copies for flight $j$ given by the set $U_{j}$. The multiple copies complicates the formulation of the CV-PSP, requiring that at most one copy $v$ for each flight $j$ is used as a reallocation option. This is enforced through the addition of the variables $w_{i j}^{v}$ and constraints (26).

A more convenient formulation of the cancellation variable subproblem can be found by eliminating the need to consider multiple copies for each flight. This is achieved for the CV-PSP by identifying a single copy $v^{\prime}$ for each flight $j$ that satisfies $\lambda_{j}^{v^{\prime}}=\min _{v \in U_{j}}\left\{\lambda_{j}^{v}\right\}$. Replacing $\sum_{v \in U_{j}} \lambda_{j}^{u}$ with $\lambda_{j}^{v^{\prime}}$ and setting $w_{i j}^{v^{\prime}}=1$ for all flights $j \in N_{i}^{\text {post }}$ forces the use of at most one copy for each flight. The resulting problem is called the reduced pricing subproblem (CV-PSPR), which is a bounded knapsack problem that is equivalent to the CV-PSP. The CV-PSPR is given by, 


$$
\begin{aligned}
\tilde{d}_{i p}=\max & \sum_{j \in N_{i}^{\text {post }}} h_{i j p}^{v^{\prime}}\left(g^{C A N}-\lambda_{j}^{v^{\prime}}-g_{i j}^{R A}\right), \\
\text { s.t. } & \sum_{j \in N_{i}^{\text {post }}} h_{i j p}^{v^{\prime}} \leq \operatorname{Pax}(i), \\
& h_{i j p}^{v^{\prime}} \leq r_{j} \quad \forall j \in N_{i}^{\text {post }}, \\
& h_{i j p}^{v^{\prime}} \in \mathbb{Z}^{+} \quad \forall j \in N_{i}^{\text {post }} .
\end{aligned}
$$

The following theorem will prove that the optimal solution to the CV-PSPR is also optimal for the CV-PSP.

Theorem 3.1.1. The optimal solutions to the CV-PSP and CV-PSPR are identical.

Proof. Assume that an optimal solution to CV-PSP for a given flight $i$ has been found describing passenger reallocation scheme $p$ with an objective value $\tilde{d}_{i p}^{\prime}$. The optimal solution is described by the variables $\bar{h}_{i j p}^{v^{\prime}}>0, \forall j \in N_{i}^{\text {post }}$, where $v^{\prime}$ is the copy for flight $j$ where $w_{i j}^{v^{\prime}}=1$.

Let $\exists j_{0} \in N_{i}^{\text {post }}$, such that $\lambda_{j_{0}}^{v^{\prime}}>\min _{v \in U_{j_{0}}}\left\{\lambda_{j_{0}}^{v}\right\}$, and $\forall j \in N_{i}^{\text {post }} \backslash j_{0}, \lambda_{j}^{v^{\prime}}=\min _{v \in U_{j}}\left\{\lambda_{j}^{v}\right\}$. Therefore, the objective value for the optimal solution to the CV-PSP is can be written as,

$$
\tilde{d}_{i p}^{\prime}=\sum_{j \in N_{i}^{\text {post }} \backslash j_{0}} \bar{h}_{i j p}^{v^{\prime}}\left(g^{C A N}-\lambda_{j}^{v^{\prime}}-g_{i j}^{R A}\right)+\bar{h}_{i j_{0} p}^{v^{\prime}}\left(g^{C A N}-\lambda_{j_{0}}^{v^{\prime}}-g_{i j_{0}}^{R A}\right) .
$$

Since $\bar{h}_{i j_{0} p}^{v^{\prime}}>0 \Rightarrow \sum_{v \in U_{j_{0}} \backslash v^{\prime}} \bar{h}_{i j_{0} p}^{v^{\prime}}=0$. Let $\lambda_{j_{0}}^{v^{\prime \prime}}=\min _{v \in U_{j_{0}}}\left\{\lambda_{j_{0}}^{v}\right\}$ and since $r_{j_{0}}$ does not depend on $v \in U_{j_{0}}$ another feasible solution to CV-PSP is given by setting $\hat{h}_{i j p}^{v}=\bar{h}_{i j p}^{v}, \forall j \in N_{i}^{\text {post }}, \forall v \in U_{j}$ with $\hat{h}_{i j_{0} p}^{v^{\prime \prime}}=\bar{h}_{i j_{0} p}^{v^{\prime}}$ and $\hat{h}_{i j_{0} p}^{v^{\prime}}=0$.

Now $\lambda_{j_{0}}^{v^{\prime \prime}}<\lambda_{j_{0}}^{v^{\prime}}$, so the objective value for this new feasible solution $\tilde{d}_{i p}^{\prime \prime}$ is greater than $\tilde{d}_{i p}^{\prime}$. Hence, $\tilde{d}_{i p}^{\prime}$ is not the objective value for the optimal solution to the CV-PSP, which is a contradiction to the original assumption.

Therefore, the optimal solution to the CV-PSP exists only when $\lambda_{j}^{v^{\prime}}=\min _{v \in U_{j}}\left\{\lambda_{j}^{v}\right\}, \forall j \in N_{i}^{\text {post }}$, which is the objective value given by the solution to the CV-PSPR. So the optimal solutions to the CV-PSP and CV-PSPR are identical.

The CV-PSPR describes a bounded knapsack problem, hence a greedy heuristic solves the linear relaxation to optimality. Additionally, the right hand side of constraints (30) and (31) are integer, so the solution to the greedy heuristic also provides the integer optimal solution.

Scaling of the passenger reallocation constraint coefficients. The passenger reallocation constraints (11) can be likened to big-M constraints, which are well known for negatively affecting the ability to identify integer solutions. A method identified to improve the computational performance of the IRP-PR is to scale the passenger reallocation constraints (11) by $r_{j}$. By applying this scaling, the 
set of constraints (11) can be restated as,

$$
\sum_{k \in K} \sum_{p \in P^{k}} a_{j p}^{k v} x_{p}^{k}-\kappa_{j}^{v+}-\sum_{i \in N^{j}} \sum_{p \in P^{i}} \tilde{h}_{i j p}^{v} z_{i p} \geq 0 \quad \forall j \in N^{D}, \forall v \in U_{j}
$$

where $\tilde{h}_{i j p}^{v}=h_{i j p}^{v} / r_{j}$. Replacing constraints (11) with the scaled constraints (34) ensures that the coefficients of the crew duty variables are one in all rows of the IRP-PR. This replacement requires a modification to the reduced cost functions for the crew duty and cancellation variables. Additionally, this constraint modification significantly affects the structure of the column generation subproblem for the cancellation variables. In particular, the scaled coefficients $\tilde{h}_{i j p}^{v}$ are no longer integer, therefore integrality is not required for the decision variables. The scaled reduced cancellation variable pricing subproblem (CV-PSPRS) is defined as,

$$
\begin{aligned}
\tilde{d}_{i p}=\max & \sum_{j \in N_{i}^{\text {post }}} \tilde{h}_{i j p}^{v^{\prime}}\left(\left(g^{C A N}-g_{i j}^{R A}\right) r_{j}-\lambda_{j}^{v^{\prime}}\right), \\
\text { s.t. } & \sum_{j \in N_{i}^{\text {post }}} \tilde{h}_{i j p}^{v^{\prime}} r_{j} \leq \operatorname{Pax}(i), \\
& \tilde{h}_{i j p}^{v^{\prime}} \in[0,1] \quad \forall j \in N_{i}^{\text {post }} .
\end{aligned}
$$

Since the CV-PSPRS is also a bounded knapsack problems formulated as a linear program, the optimal solution is found using a greedy heuristic. Similar to the CV-PSPR, the right hand side of constraints (36) are all integer, hence only integer quantities of passengers are reallocated to each available flight. The computational experiments presented in Section 4 will discuss the improvements in the solution runtimes for the IRP-PR achieved by applying the described scaling of the coefficients for the cancellation variables.

\subsection{Row generation}

The full column-and-row generation solution approach is described by Algorithm 3 in Maher [20]. The following sections will describe the application of the key steps of Algorithm 3 to the IRP-PR. These steps include, i) the formulation of the restricted problem, ii) computing an optimal dual solution, and iii) the addition of rows to update the restricted problem.

\subsubsection{Formulation of the restricted problems}

Modelling flight delays using flight copies is convenient regarding the solution algorithms for the column generation subproblem; however, an unfortunate consequence is the large number of related constraints in the RMP. This is a result of the number of the delay consistency (10) and passenger reallocation constraints (11) being directly proportional to the number of flight copies.

The short restricted master problem (SRMP) is defined as a further restriction of the RMP by eliminating a set of structural constraints. The SRMP is formulated for the IRP-PR to including only 
a subset of all possible rows from the delay consistency (10) and passenger reallocation constraints (11) that form the RMP. Since the optimal integer solution to the IRP-PR selects exactly one delay copy $v^{\prime}$ for each flight $j$, it would be ideal to formulate the SRMP with $U_{j}=\left\{v^{\prime}\right\}, \forall j \in N^{D}$. While it is not possible to identify $v^{\prime}, \forall j \in N^{D}$ in the construction of the IRP-PR, the SRMP is formed with the set $\bar{U}_{j} \subseteq U_{j}$. The set $\bar{U}_{j}$ provides an initial guess at the optimal formulation of the IRP-PR which is progressively updated by a row generation procedure. The elimination of rows to form the SRMP results in a problem that can be solved by column generation using the methods described in Section 3.1.

The sets $\bar{U}_{j}$ are initialised for each flight $j$ by including the copy that represents the scheduled departure time, i.e. $\bar{U}_{j}=\{0\}$. However, it is likely due to the delays associated with the disruption scenarios that for some flights $j^{\prime}$ there exists no feasible connection containing the flight-copy $j_{0}^{\prime}$. In these situations, an additional copy $v^{\prime}$ is included in the set $\bar{U}_{j}$, such that $j_{v^{\prime}}^{\prime}$ is the flight-copy pair that provides at least one feasible connection and the smallest amount of delay for $j^{\prime}$.

A key feature of column-and-row generation is the fixing of variables in the column generation subproblems as a result of eliminating constraints. The variable fixings in the column generation subproblems ensure that all generated flight string and reallocation schemes have zero coefficients in the eliminated rows. The elimination of rows and the related variable fixings reduce the size of the column generation master and subproblems respectively, having a positive effect on the solution runtimes. In addition, it permits the property that the solution to the SRMP is an upper bound on the RMP and the IRP-PR.

\subsubsection{Row generation algorithm}

The row generation procedure is described by Algorithm 2 in Maher [20]. This algorithm involves two major stages, the calculation of the optimal dual solution and identifying favourable rows to include in the SRMP.

In Maher [20], the $\mathrm{RMP}^{\prime}$ is constructed to contain all rows from the RMP and all variables from the SRMP. In addition, a set of artificial variables are introduced to populate the rows in the $\mathrm{RMP}^{\prime}$ that are eliminated to form the SRMP. The construction of the $\mathrm{RMP}^{\prime}$ is critical in the row generation procedure, facilitating the calculation of an optimal dual solution using the solution to the SRMP.

The rows contained in the $\mathrm{RMP}^{\prime}$ are partitioned into two groups, those common and uncommon with the SRMP. For the rows common between the RMP' and SRMP, the dual solutions can be simply equated. A procedure is then executed to calculate the dual solutions for the rows uncommon between the two problems.

The dual variables for the rows related to $U_{j} \backslash \bar{U}_{j}$ for the two secondary variables in the $\mathrm{RMP}^{\prime}$ are given by $\gamma^{\prime}=\left\{\gamma_{j}^{v^{\prime}}, \forall j \in N^{D}, \forall v^{\prime} \in U_{j} \backslash \bar{U}_{j}\right\}$ and $\boldsymbol{\lambda}^{\prime}=\left\{\lambda_{j}^{v^{\prime}}, \forall j \in N^{D}, \forall v^{\prime} \in U_{j} \backslash \bar{U}_{j}\right\}$. Since the dual variables contained in $\gamma^{\prime}$ and $\boldsymbol{\lambda}^{\prime}$ are related to the rows not included in the SRMP, the solutions are calculated using the $\mathrm{PSP}^{R}$ and CV-PSPRS respectively in step 2 of Algorithm 1 [20]. Similar 
to [20], the $\mathrm{PSP}^{R}$ is used as a problem specific enhancement to reduce the time required for the row generation procedure. Unfortunately, no such general column generation subproblem can be formed for the cancellation variables, requiring the CV-PSPRS to be solved once for each $i \in N^{j}$ to calculate $\lambda_{j}^{v^{\prime}} \in \boldsymbol{\lambda}^{\prime}$.

Favourable rows for the SRMP are identified by evaluating the reduced costs of primary variables feasible for the RMP'. For the IRP-PR, the primary variables are the crew duty variables, as such problem (18) is solved in step 8 of Algorithm 2 [20]. To identify crew duty variables feasible for the $\mathrm{RMP}^{\prime}$, problem (18) is solved with all possible delay copies given by $U_{j}$. If any negative reduced cost variables are found, the flight-copy pairs contained on the related flight strings indicate the rows that must be included in the SRMP. In addition to adding rows to the SRMP, the related variable fixings in the column generation subproblems are relaxed, increasing the size of the connection network. If no negative reduced cost variables are found, the current solution for the SRMP is the optimal solution to the IRP-PR. In an effort to improve the solution runtimes of the row generation procedure, a greater number of rows to add to the SRMP are identified by solving problem (18) once for each $k \in K$. This is a problem specific enhancement technique that is also employed in Maher [20].

A number of enhancement techniques for column-and-row generation are developed and evaluated in Maher [20]. In particular, a method to increase the number of rows added to the SRMP and a row warm-up procedure are discussed. The presented results demonstrate improved runtime performance from the implementation of these techniques, as such they are also employed in the solution process of the IRP-PR. In the following section an additional enhancement technique is described that uses properties of the row generation framework to improve the convergence of the branch-and-price algorithm.

\subsection{Variable fixing}

The feasible regions of the network flow problems $\mathrm{PSP}^{k}$ and $\mathrm{PSP}^{r}$ conveniently describe the variables contained in $P^{k}$ and $P^{r}$ respectively. Such a network flow problem is formulated with arc-based variables $w_{i_{u} j_{v} p}^{k}$ for crew $\left(w_{i_{u} j_{v} p}^{r}\right.$ for aircraft) that equal 1 to indicate that connection $\left(i_{u}, j_{v}\right)$ is used on flight string $p$. The elimination of rows to form the SRMP is coupled with the fixing of arc-based variables $w_{i_{u} j_{v} p}^{k}=0\left(w_{i_{u} j_{v} p}^{r}=0\right)$ in the network flow problems, where $v \in U_{j} \backslash \bar{U}_{j}$. This variable fixing can also be described as the elimination of connections from the networks used to solve the shortest path problems for crew and aircraft. As a result, the runtimes required to solve the column generation subproblems for the SRMP are much shorter than that for the RMP.

The row generation procedure described in Section 3.2.2 involves the addition of rows to the SRMP and the unfixing of variables in the column generation subproblems. This causes the size of the connection networks used for each shortest path problem to increase, having a negative effect on solution runtimes. A variable fixing heuristic is proposed in this section that reduces the size of the column generation subproblems using the optimal solution to the SRMP at the root node of the branch-and-bound tree. 
This heuristic assumes that the flight-copy pairs used in the optimal solution of the LP relaxation for the SRMP have a high probability of occurring in the integer optimal solution. Given the high level of degeneracy in airline planning and recovery problems, it is also assumed that the flight-copy pairs included on variables that have a reduced cost of zero may be used in the integer optimal solution. More formally, the set $I=\left\{j_{v} \mid \bar{c}_{p}^{k}=0 \wedge a_{j p}^{k v}=1, \forall k \in K, \forall p \in P^{k}\right\}$ defines the set of flight-copy pairs that are expected to be included on basic variable flights strings. This identifies a subset of flight-copy pairs that can be used to eliminate rows from the SRMP and reduce the size of the column generation subproblems. From $I$, the set of connections included in the column generation subproblems is defined as $\bar{C}=\left\{\left(i_{u}, j_{v}\right) \mid i_{u} \in I \wedge j_{v} \in I\right\}$, hence the variable fixing, $w_{i_{u} j_{v}}^{k}=0, w_{i_{u} j_{v}}^{r}=0, \forall\left(i_{u}, j_{v}\right) \notin \bar{C}$ can be applied.

This heuristic uses information from the optimal solution to the LP relaxation of the IRP-PR as a proxy for the integer optimal solution. As such, it is not guaranteed that the solutions providing the best lower and upper bounds for the IRP-PR will contain only the flight-copy pairs included in I. Consequently, it is possible that the integer optimal solution that is found using this heuristic will overestimate the true optimal solution. To provide a meaningful comparison of the solution quality by column generation and column-and-row generation, the optimality gap in this paper is calculated using a lower bound set at the LP solution of the root node.

\section{Computational Results}

There are two distinct aims of the computational results presented in this section, i) demonstrate the benefits of considering passengers in the integrated recovery process, and ii) provide an analysis of the general column-and-row generation framework presented in Maher [20]. The modelling of passenger recovery in the IRP-PR involves the reallocation of passengers to alternative flights when flight cancellations are required as part of the recovery process. The benefits from considering this passenger recovery approach is assessed against the IRP from [20], with a comparison made in regards to runtime, cost and disruption statistics. At present, the only evaluation of the general column-and-row generation framework presented in Maher [20] involves solving a simplified model with a single set of secondary variables and linking constraints. The IRP-PR is a much more complex problem, as such the computational results will provide a more in-depth analysis of the the framework developed in [20].

The measure of solution quality is based upon the optimality gap achieved at the termination of the maximum allowable runtime. This measure is important for the scenarios that fail to solve within this runtime, requiring the best found integer solution to be implemented. In Section 3.3, a variable fixing heuristic is introduced which potentially overestimates the lower bound of the IRP-PR. As stated, a meaningful comparison between the column generation and column-and-row generation solution approaches is provided by calculating the optimality gap as the relative difference between the LP solution 
at the root node and the best found integer solution. Any reference to the optimality gap hereafter will represent the gap calculated in this manner.

\subsection{Description of data and disruption scenarios}

The data and disruption scenarios that are used for this problem are identical to those related to the point-to-point flight schedule presented in Maher [20]. The motivation for using the same point-to-point schedule is to provide consistency in the analysis of the two different recovery models. Therefore, only a brief description of the key features will be provided in this section.

The IRP and IRP-PR are evaluated using a single day flight schedule that contains 262 flights transporting 28,492 passengers, which are serviced by 48 aircraft and $79 \mathrm{crew}$ groups. The flight schedule services 20 airports, of which 12 are overnight bases for aircraft and 4 are crew bases. Of the 20 airports, the majority of flights originate or terminate at only 2. A set of 16 disruption scenarios are generated for the evaluation of the IRP and IRP-PR, each representing a closure at one of the major airports starting at $6 \mathrm{am}, 7 \mathrm{am}, 8 \mathrm{am}$ or $9 \mathrm{am}$ and lasting for a total duration of 3 or 5 hours. Airport closure scenarios are selected for evaluation purposes due to the significant associated recovery costs. Throughout this section the scenarios will be referred to by an identifying number as detailed in Table 1.

\begin{tabular}{|c|cccc|}
\hline Start Time & 6am & 7am & 8am & 9am \\
\hline Airport 1 & $(0,8)$ & $(2,10)$ & $(4,12)$ & $(6,14)$ \\
Airport 2 & $(1,9)$ & $(3,11)$ & $(5,13)$ & $(7,15)$ \\
\hline
\end{tabular}

Table 1: Scenario numbers used in the presentation of results. The bracketed values indicate two different closure durations (3 hours, 5 hours).

A full set of recovery policies, including the generation of new aircraft routes and crew duties, flight delays and cancellations, crew deadheading and the use of reserve crew are implemented for the IRP and IRP-PR. The recovery actions are permissible directly after the disruption occurs until the end of a selected recovery window. For these experiments the recovery window is set at 6 hours, commencing after the affected airport is reopened. While the use of a recovery window approximates the complete recovery problem, this approach is consistent with the objective to return operations back to plan as quickly as possible. The number of flights that depart within the closure period and recovery window is documented in Table 2. It is important to note that while the number of included flights is reduced, no approximation is made to reduce the number of affected crew and aircraft.

\begin{tabular}{|l|cccccccccccccccc|}
\hline Scenario & 0 & 1 & 2 & 3 & 4 & 5 & 6 & 7 & 8 & 9 & 10 & 11 & 12 & 13 & 14 & 15 \\
\hline$\left|N^{D}\right|$ & 150 & 151 & 149 & 150 & 147 & 145 & 150 & 149 & 182 & 183 & 185 & 186 & 184 & 182 & 184 & 183 \\
\hline
\end{tabular}

Table 2: The number of disruptable flights for each scenario.

Flight delays and cancellations are two of the most effective actions that are available to airlines in 
the recovery from disruptive events. Flight delays have been modelled using the common flight copies technique, with 7 copies used for the IRP and IRP-PR to discretise a maximum delay of 180 minutes. Attempting to provide a true cost of delays, the estimate detailed in the EUROCONTROL report by Cook and Tanner [14] has been used in these experiments. In [14] the cost of delaying a full aircraft for a minute is estimated at $\$ 100$ AUD.

The cost of cancelling a flight in the IRP-PR accounts for the number of passengers reallocated to operating flights. If a passenger is rebooked onto another flight operated by the airline, the full revenue is retained minus any delay costs associated with waiting for a later departure time. It is common that the number of available flying seats is less than the number of passengers requiring reallocation, as such some passengers are not provided alternative arrangements resulting in the full loss of ticket revenue for the airline.

The IRP solves a recovery problem integrating aircraft and crew to fit within the commonly applied sequential recovery process. As part of this process, passenger recovery is undertaken after all preceding stages have been solved. To directly compare the impact of considering passenger reallocation in the integrated recovery problem, as in the IRP-PR, this reallocation process is undertaken a posteriori using the solution to the IRP. This is achieved by employing a greedy approach to rebook passengers onto the next available alternative flights, which are defined as having the same O-D pair and departing within a maximum allowable delay window.

This model is implemented in $\mathrm{C}++$ calling SCIP 3.0.1 [4] to solve the integer program using CPLEX 12.4 as the linear programming solver.

\subsection{Improvement in recovery achieved with passenger reallocation}

The analysis of the IRP and IRP-PR aims to identify the effect of modelling passenger reallocation has on the recovery costs, the recovery actions taken and the solution runtime. The results are produced by solving the two recovery models against the set of 16 scenarios detailed in Table 1 . The optimal solution to each of the models is identified when the relative difference between the primal and dual bounds is less than $5 \%$.

\subsubsection{Recovery costs}

The motivation for considering the alternative passenger recovery model given by the IRP-PR is to reduce the recovery costs incurred while accommodating disrupted passengers. The relative difference in the recovery costs between the IRP and IRP-PR is given in Figure 1. This figure demonstrates that a significant reduction in the operating costs of an airline can be realised by employing this alternative modelling technique. On average, using the IRP-PR to solve the recovery problem reduces the operational costs of the airline by $22.98 \%$. 


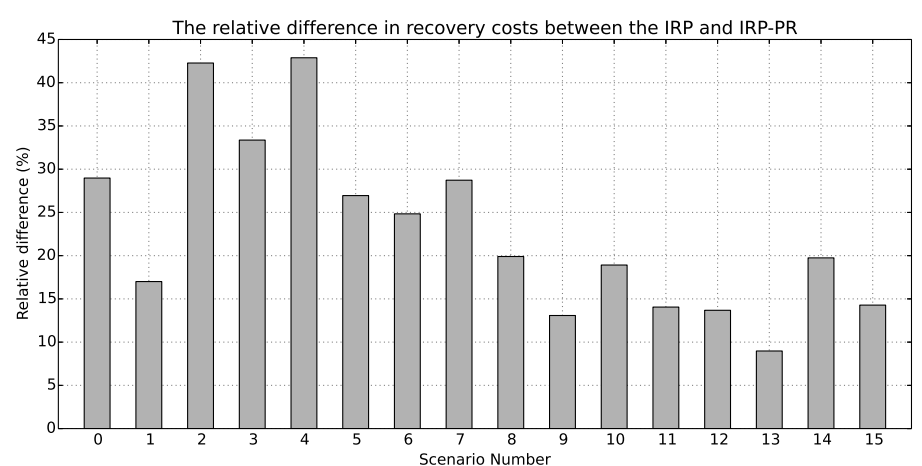

Figure 1: The relative difference between the recovery costs of the $\operatorname{IRP}(x)$ and IRP-PR $(y)$. The value of the bars is given by $(x-y) / x$.

The largest relative improvement in recovery costs is $42.89 \%$, which is achieved in scenario 4 . Comparing the solution for this scenario given by the two alternative recovery models, there is a marked difference in the number of flight cancellations. Specifically the IRP cancels 5 flights compared to 10 for the IRP-PR. Now, this increase in the number of cancelled flights achieves a smaller recovery cost for the solution to the IRP-PR since all except 27 passengers are provided alternative travel arrangements. By contrast, the greedy reallocation approach applied using the solution to the IRP leaves 380 passengers stranded by the airline. The number of passengers stranded following a flight cancellation is greater than the total number of seats on a single flight. This demonstrates the magnitude of the disruption experienced by passengers as a result of solving the IRP. The ability of the IRP-PR to provide alternative travel arrangements for the majority of passengers on cancelled flight is common across all scenarios. Therefore, the consideration of passenger reallocation allows the strategic cancellation of flights to reduce operational costs of the airline.

\subsubsection{Cancellation and delay information}

Considering passengers in the recovery process aims to reduce the impact of cancellations and delays on passenger travel arrangements. In particular, directly modelling reallocation options following flight cancellations ensures that passengers are routed to their destination with minimal delay. The reduction in recovery costs, presented in Section 4.2.1, benefits the airline, however this does not necessarily imply improved passenger satisfaction. For the analysis presented in this section it is assumed that providing passengers with alternative travel arrangements directly improves passenger satisfaction.

Figure 2 presents the total number of disrupted passengers and the effect of the disruption on their travel arrangements. The bars are divided into three different groups, i) the reallocated group are the passengers on cancelled flights that are rebooked onto alternative operating flights, ii) the delayed group includes all passengers that are booked on flights that depart later than scheduled, and iii) the cancelled group are the passengers for which no alternative travel arrangements are provided. For all passengers in the cancelled group, the tickets are refunded and the passengers must rebook themselves onto alternative 


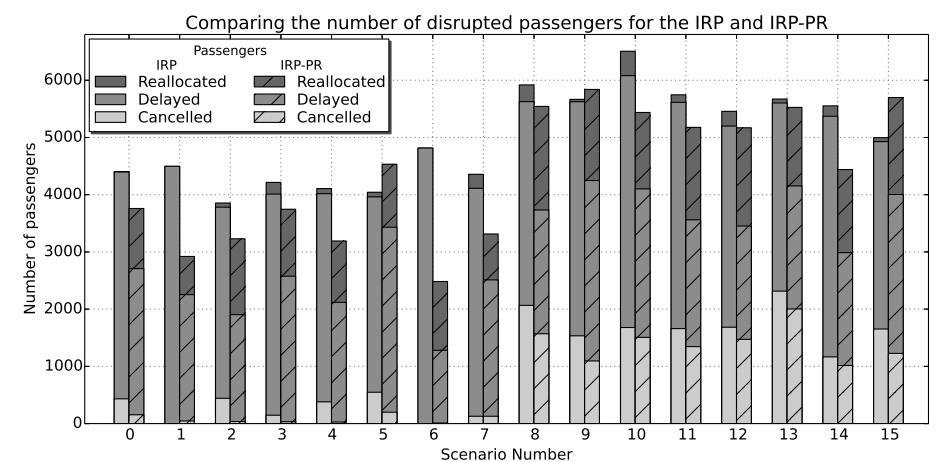

Figure 2: The number of reallocated, delayed and cancelled passengers from the IRP (bars) and IRP-PR (bars with hatching) solutions.

flights. The passengers that are placed in this group are the most affected by the disruption, resulting in poor passenger satisfaction and the potential loss of future bookings.

The passenger reallocation approach developed for the IRP-PR increases the number of cancelled flights, however this is combined with an increase in the number of passengers reallocated onto alternative flights. Since reallocation options are directly modelled, flight delay decisions are made to ensure an adequate number of seats are available for the passengers on cancelled flights. It is clear in Figure 2 that by solving the IRP-PR more passengers are provided with alternative travel arrangements compared to the IRP, greatly reducing the number of cancelled passengers in each scenario. This result has a direct positive impact on passenger satisfaction by ensuring that a greater number of passenger arrive at their destination within a reasonable time frame. The average on-time performance of the airline is very similar using the solutions to the IRP and the IRP-PR. The major difference between the two models is improved flow of passengers through the network achieved by solving the IRP-PR. On average, the solution to the IRP-PR disrupts 4376 passengers compared to 4988 for the IRP, providing a decrease of 612 passengers. Given that the solution to the IRP-PR provides a smaller recovery cost than the IRP, the decrease in the number of disrupted passengers demonstrates that this cost saving is not at the expense of passengers.

\subsubsection{Solution runtimes}

The additional variables and constraints related to passenger reallocation decisions in the IRP-PR increases the problem complexity compared to the IRP. As a result, it is expected that longer runtimes are required to identify the optimal integer solution for the IRP-PR. This is evident in Figure 3 where the IRP is solved to optimality faster than the IRP-PR for 11 of the presented scenarios. The main difference between the solution process for each model is that the IRP-PR generally requires a greater number of branches to converge to the integer optimal solution. Across all scenarios, the IRP-PR requires 66.25 nodes on average compared to 8.56 for the IRP. This is a significant difference in the solution process that has a great effect on the algorithm runtimes. 


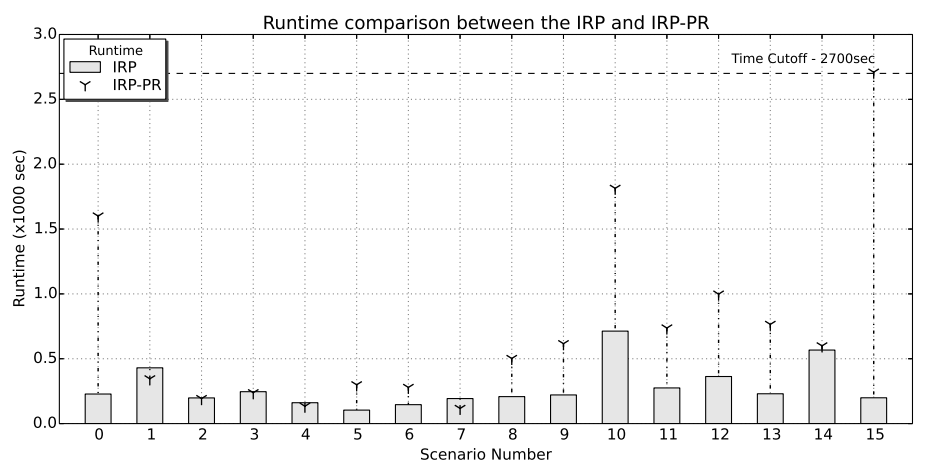

Figure 3: The runtimes to solve the IRP (bars) and IRP-PR (stars) using column-and-row generation for each scenario with a maximum of 2700 seconds.

The slow convergence displayed by the IRP-PR can be explained further by analysing the optimality gap of the two models at the root node, which presented in Table 3. This table shows that 4 of the scenarios are solved to optimality by the LP solution at the root node for the IRP, where this does not occur in any scenario for the IRP-PR. In addition, the optimality gap for the IRP-PR is significantly larger than that for the IRP in all but one scenario, indicating the potential requirement of a greater number of branches in the branch-and-price algorithm. This is to be expected since the passenger reallocation constraints (11) are in the form of big-M constraints, which commonly display difficulties in identifying integer solutions.

\begin{tabular}{|l|cccccccc|}
\hline Scenario & 0 & 1 & 2 & 3 & 4 & 5 & 6 & 7 \\
\hline IRP & $2083.49 \%$ & $5305.47 \%$ & $1825.2 \%$ & $2957.86 \%$ & $1735.34 \%$ & $0.0 \%$ & $0.0 \%$ & $2276.56 \%$ \\
IRP-PR & $3558.8 \%$ & $6199.04 \%$ & $4462.38 \%$ & $5407.34 \%$ & $4241.88 \%$ & $3071.69 \%$ & $3969.75 \%$ & $4976.35 \%$ \\
\hline \hline Scenario & 8 & 9 & 10 & 11 & 12 & 13 & 14 & 15 \\
\hline IRP & $872.49 \%$ & $0.0 \%$ & $1201.56 \%$ & $1238.49 \%$ & $1469.05 \%$ & $0.0 \%$ & $3354.78 \%$ & $3547.17 \%$ \\
IRP-PR & $1392.51 \%$ & $1916.24 \%$ & $1654.11 \%$ & $2195.46 \%$ & $2459.03 \%$ & $1516.11 \%$ & $5755.05 \%$ & $4772.27 \%$ \\
\hline
\end{tabular}

Table 3: The optimality gap at the root node from solving the IRP and the IRP-PR.

The runtime comparison between the IRP and the IRP-PR demonstrate that the increased complexity introduced by the passenger reallocation decisions increases the solution runtime. While this increase in solution runtimes is not ideal for an airline recovery problem, the difference is not prohibitively large. Figure 3 demonstrates that the vast majority of scenarios for the IRP-PR are solved within 1200 seconds (20 minutes), which is within an acceptable runtime for practical use of the algorithm. Comparing Figures 1 and 3 , it can be concluded that the potential gains from included passenger reallocation outweigh the resulting increase in runtime.

\subsection{Evaluation of modelling approaches}

The IRP-PR incorporates a number of modelling approaches to improve robustness and maintain the consistency of the recovered solution. In particular, short connection constraints force crew and aircraft 
to operate the same connections that increases the permissible set of connections and a bonus parameter is introduced that reduces the number of changes between the planned and recovered solutions. Both of these approaches, while beneficial in an operations context, can result in suboptimal recovered solutions. This section demonstrates the effect that implementing each of these approaches has on the optimal recovered solution.

\subsubsection{Effect of short connection constraints}

Short connection constraints aim to improve the efficiency of the planned solution by permitting shorter ground times for crew. It is common for scheduling decisions to be made by considering such "short connections", especially at smaller airports in the network, hence it may be necessary to include a subset of constraints (9) in the recovery problem formulation. To avoid causing infeasibilities by omitting short connection constraints, those required as a result of scheduling decisions are still included in the problem formulation.

Figure 1 presents the relative difference in the recovery costs from solving the IRP-PR with and without the short connection constraints. Since the formulation of the IRP-PR given by (1)-(17) includes the short connection constraints, this is used as the base case. The results in Figure 4 demonstrate significant increases in the optimal recovery costs as a result of omitting the short connection constraints from the problem formulation. This is not surprising, since the omission of the short connection constraints reduces the number of available connections for crew in the recovered solution. So, including short connection constraints in the IRP-PR improves the robustness of the resulting solution and reduces the overall costs.

The largest relative difference in recovery costs is given by scenario 10 , which is due to the model failing to identify a good optimality gap in the allocated runtime. Additionally, it is observed for the larger scenarios $(8-15)$ that omitting the short connection constraints from the IRP-PR results in an increase to the solution runtimes. In this set of scenarios, the time limit is reached for four and there

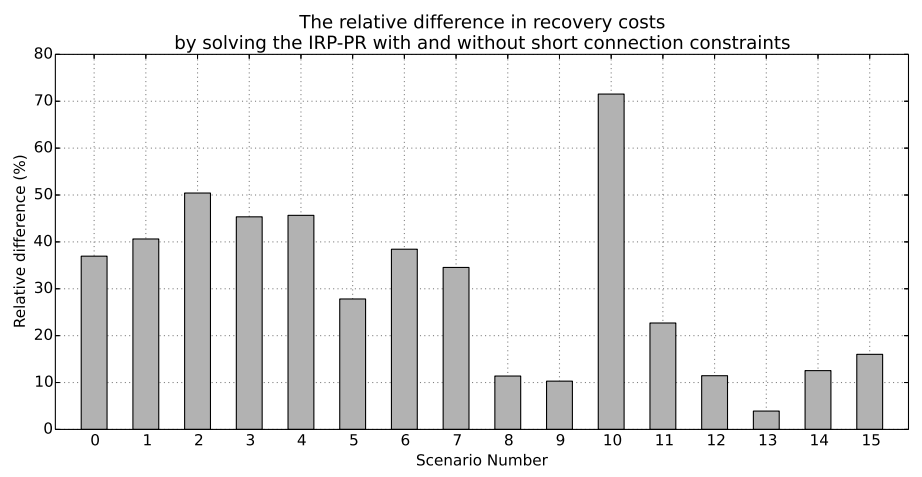

Figure 4: The relative difference between the standard implementation of the IRP-PR (with short connection constraints) $(x)$ and the IRP-PR without short connection constraints $(y)$. The value of the bars is given by $(y-x) / y$. 
is only one where the runtime is shorter than for the standard implementation of the IRP-PR. This suggests that the inclusion of the short connection constraints forms a better LP relaxation, reducing the time required to identify the optimal integer solution.

\subsubsection{Effect of the bonus parameter}

Solving the IRP-PR identifies a set of crew pairings and aircraft routes to operate on the perturbed flight schedule. As a result of the schedule perturbations, the generated pairings and routes may be vastly different to those constructed in the planning stage. This situation, while providing the optimal solution, may present difficulties for the operations controllers in the practical implementation the provided solution. Hence, it is assumed that introducing a bonus parameter, however small, to reduce the number of changes that are made during recovery may be beneficial.

The results presented in Section 4 involve solving the IRP-PR using a bonus parameter of 0.01. This small bonus is used to prevent the generation of a completely new set of pairings and routes, but still permitting sufficient flexibility to achieve a recovered solution that is close to optimal. Figure 5 presents the relative difference in the recovery costs between the base case and that from applying bonuses greater than 0.01 .

The results presented in Figure 5 demonstrate much variation with the use of different parameters across the set of scenarios. However, there appears to be a general trend where solving the IRP-PR with larger bonus parameters, i.e. 100 and 1000, results in a recovery cost increase. This is to be expected, since the use of bonus parameters reduces the flexibility of the recovery problem. There are two different effects resulting from this reduced flexibility, i) the construction of suboptimal crew pairings and aircraft routes, and ii) an increase in the number of delayed and cancelled flights. The first of these effects is observed in scenario 1, where the cost of crew increases substantially as a result of increases in the bonus parameter. This indicates that the recovered solution for scenario 1 forces the crew to work longer hours than originally planned. Since the crew costs increase, little change in the number of delayed and

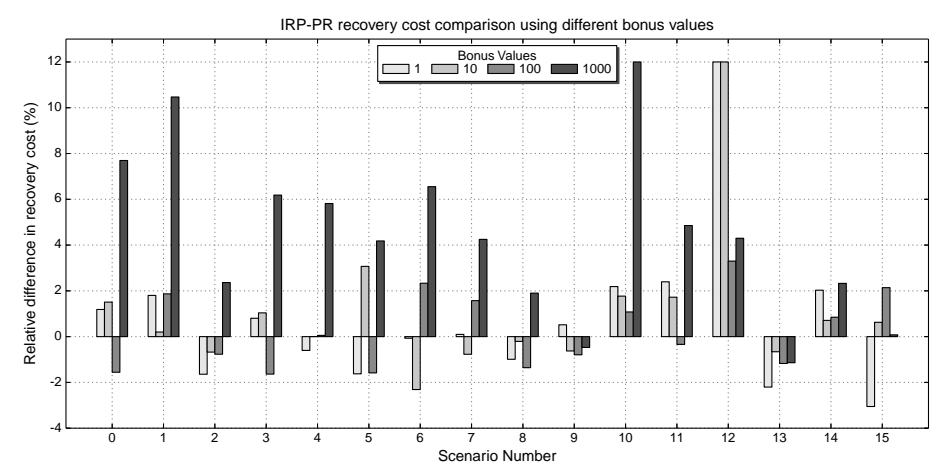

Figure 5: The relative difference in the recovery costs between solving the IRP-PR with a bonus of 0.01 $(x)$ and bonuses of $1,10,100$, and $1000(y)$. The values in the figure are calculated by $(y-x) / x$. For improved presentation the maximum relative difference is capped at $12 \%$. 
cancelled flights are observed. Alternatively, scenario 6 demonstrates a decrease in the crew costs as the bonus parameter increases but an increase in the number of delay minutes. This increase in delay minutes is a consequence of the model attempting to construct crew pairings and aircraft routes without much deviation from the planned solution. Hence, the main recovery policy available is flight delays.

\subsection{Evaluation of column-and-row generation}

A general column-and-row generation framework to solve problems with multiple secondary variables and linking constraints is developed and evaluated in Maher [20]. However, the IRP presented in [20] is a special case of the problems to which the column-and-row generation framework can be applied. Since the IRP-PR is modelled with two sets of secondary variables and linking constraints, this paper presents an evaluation of the column-and-row generation framework against a more general problem formulation. The following sections provide a comparison between standard column generation and column-and-row generation and the assessment of enhancement techniques.

\subsubsection{Comparison between column generation and column-and-row generation}

The runtime required to solve the IRP-PR using column generation and column-and-row generation is presented Figure 6. This figure demonstrates a reduction in solution runtimes for the IRP-PR when solved with column-and-row generation. These result are encouraging since it supports the results presented in Maher [20]. This suggests that the general column-and-row generation framework is also very effective on problems with multiple secondary variables and linking constraints.

The runtime results in Figure 6 indicate that a higher quality solution is achievable using columnand-row generation compared to column generation with shorter runtimes. The runtimes presented in Figure 6 show that column-and-row generation outperforms column generation in 12 of the presented experiments. This provides a relative improvement in solution runtimes of $24.35 \%$, significantly improving

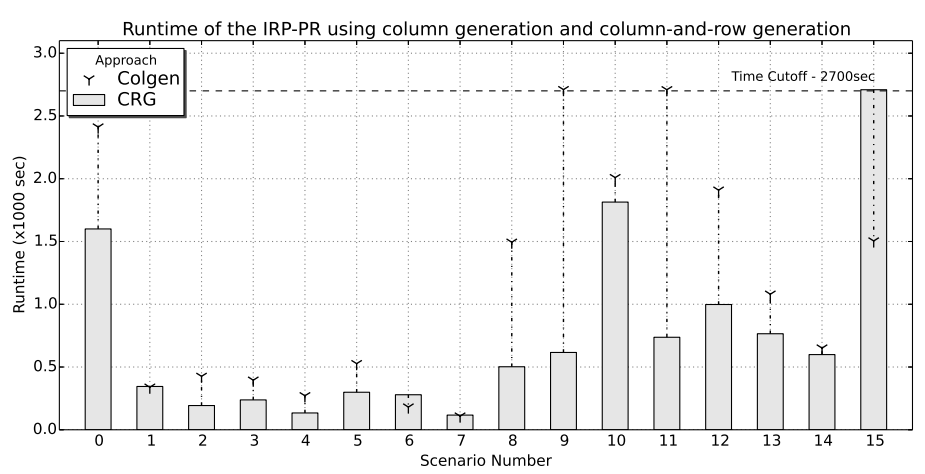

Figure 6: The runtimes to solve the IRP-PR for each scenario with a maximum of 2700 seconds. This figure compares the solution approaches of column-and-row generation (bars) and column generation (stars). 
upon the results given by Maher [20]. It is clear through the explicit evaluation of the solution runtimes, column-and-row generation significantly outperforms a standard column generation approach.

\subsubsection{Runtime enhancement techniques}

A number of enhancements techniques designed to improve the solution runtime and quality of columnand-row generation are proposed in Section 3. In particular, a variable fixing heuristic and the reformulation of the cancellation variables have been developed specifically for the IRP-PR. However, it is possible to adapt the variable fixing heuristic to other implementations of the column-and-row generation framework.

The variable fixing heuristic restricts the allowable delay copies used to construct columns, therefore reducing the feasible region of the IRP-PR. This technique attempts to reduce the runtimes of the subproblem and LP solves in each iteration of the column generation algorithm. The modelling of the cancellation variables in this paper is a unique approach for considering passenger reallocation for the integrated recovery problem. Section 3.1.2 details the scaling of the coefficients for the crew duty and cancellation variables in the reallocation constraints (11). Since the reallocation constraints (11) are in the form of big-M constraints, this scaling to form constraints (34) is proposed to alleviate the common issues related to identifying integer solutions.

Figure 7 details the time required to solve the scenarios with the IRP-PR, implementing the variable fixing heuristic and/or the cancellation variable scaling enhancement techniques. It is interesting to note that all except one scenario is solved to optimality within the maximum runtime of 2700 seconds (45 minutes). The best runtime performance of the IRP-PR is given when the heuristic is used in isolation or both enhancements are used. As expected, the best overall runtime result is achieved when both of the enhancement techniques are implemented.

The enhancement techniques attempt to form a SRMP that is more easily solvable by branch-andprice. Comparing the frontiers created by each of the enhancement techniques, the greatest improvement

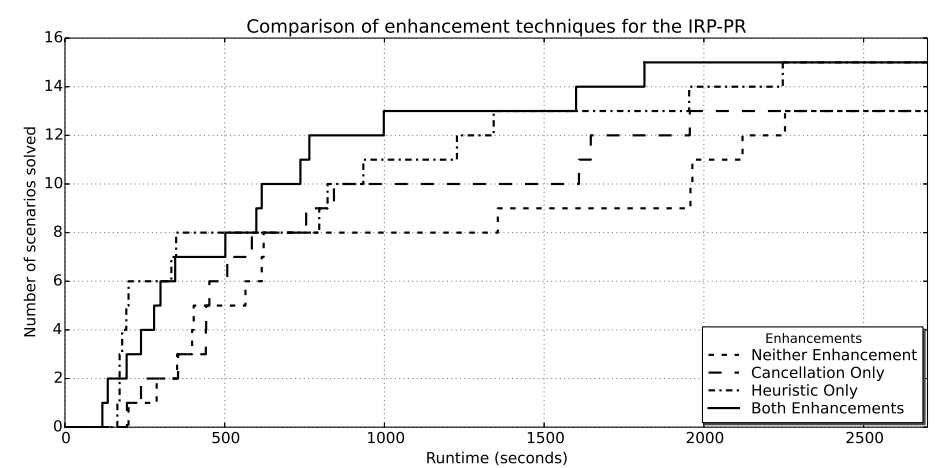

Figure 7: Time required to solve scenarios by employing different enhancement techniques, specifically the variable fixing heuristic and/or the cancellation variable scaling enhancements. A maximum runtime of 2700 seconds (45 minutes) is applied. 
is observed for scenarios requiring greater than 500 seconds runtime. Now, the average time to solve the root node when no enhancements are used is 173.25 seconds, with a maximum of 242 seconds. By comparison, the average time to solve the root node when all enhancements are employed is 164.53 seconds. This suggests that the enhancements aid in improving the convergence to the integral optimal solution for the larger scenarios.

\section{Conclusions}

This paper presents a novel approach for considering passengers in an integrated airline recovery problem through the modelling of cancellation variables. The cancellation variables have been modelled as knapsack variables to describe the possible reallocation options for passengers in the event of a flight cancellation. This modelling approach provides a simple and effective method to consider passengers in recovery. To achieve fast solution runtimes for the IRP-PR, the column-and-row generation solution approach is applied. The computational experiments presented in this chapter provides an extensive evaluation of the general column-and-row generation framework developed by Maher [20].

The passenger reallocation approach developed for the IRP-PR is demonstrated through experiments to greatly reduce the recovery costs achieved by the IRP. Further, the solution to the IRP-PR achieves a greater passenger flow through the network as a result of strategic delay decisions. The benefits achieved by solving the IRP-PR are realised by the airline and passengers through a reduction in costs and the magnitude of disruption.

The computational experiments present a comparison between column generation and column-androw generation, demonstrating significant runtime improvements achieved by the latter. In addition, a number of enhancements have been proposed and the results present a reduction in the solution runtime of the IRP-PR by improving the convergence to the integer optimal solution. The variable fixing heuristic is developed using characteristics of the column-and-row generation solution approach, as such it is possible to implement such a heuristic for similar problems where column-and-row generation is applied.

Passenger recovery in the IRP-PR is a simple and novel approach that is designed to reduce passenger dissatisfaction resulting from flight cancellations. While this modelling approach reduces the number of cancelled and disrupted passengers, it does not have a significant effect on the average delay experienced per passenger. Future work on this problem involves integrating the passenger reallocation approach into a broader passenger recovery scheme in an attempt to reduce the impact of flight delays. There is an expectation that further reductions in recovery costs and improvements in passenger satisfaction can be achieved with the greater consideration of passenger flows in airline recovery problems. 


\section{Acknowledgements}

SJM is supported by the Australian Research Council Centre of Excellence for Mathematics and Statistics of Complex Systems (MASCOS) and an Australian Postgraduate Award.

\section{References}

[1] Challenge ROADEF 2009: disruption management for commercial aviation. URL: http://challenge.roadef.org/2009/en/. Access date: 28th August 2013.

[2] A. Abdelghany, G. Ekollu, R. Narasimhan, and K. Abdelghany. A proactive crew recovery decision support tool for commercial airlines during irregular operations. Annals of Operations Research, $127(23): 309-331,2004$.

[3] K. F. Abdelghany, A. F. Abdelghany, and G. Ekollu. An integrated decision support tool for airlines schedule recovery during irregular operations. European Journal of Operational Research, 185(2):825-848, 2008.

[4] T. Achterberg. SCIP: solving constraint integer programs. Mathematical Programming Computation, 1(1):1-41, 2009.

[5] R. Ahuja, T. Magnanti, and J. Orlin. Network flows: theory, algorithms, and applications. Prentice Hall, 1993.

[6] J. Bard, G. Yu, and M. Argüello. Optimizing aircraft routings in response to groundings and delays. IIE Transactions, 33(10):931-947, 2001.

[7] C. Barnhart, N. L. Boland, L. W. Clarke, E. L. Johnson, G. L. Nemhauser, and R. G. Shenoi. Flight string models for aircraft fleeting and routing. Transportation Science, 32(3):208-220, 1998.

[8] C. Barnhart, A. Cohn, E. Johnson, D. Klabjan, G. Nemhauser, and P. Vance. Airline crew scheduling. In R. W. Hall, editor, Handbook of Transportation Science, volume 56 of International Series in Operations Research and Management Science, pages 517-560. Springer US, 2003.

[9] S. Bisaillon, J.-F. Cordeau, G. Laporte, and F. Pasin. A large neighbourhood search heuristic for the aircraft and passenger recovery problem. 4OR, 9(2):139-157, 2011.

[10] S. Bratu and C. Barnhart. Flight operations recovery: new approaches considering passenger recovery. Journal of Scheduling, 9(3):279-298, 2006.

[11] J. Cao and A. Kanafani. Real-time decision support for integration of airline flight cancellations and delays part I: mathematical formulation. Transportation Planning and Technology, 20(3):183-199, 1997. 
[12] J. Cao and A. Kanafani. Real-time decision support for integration of airline flight cancellations and delays part II: algorithm and computational experiments. Transportation Planning and Technology, 20(3):201-217, 1997.

[13] J. Clausen, A. Larsen, J. Larsen, and N. J. Rezanova. Disruption management in the airline industry-concepts, models and methods. Computers 65 Operations Research, 37(5):809-821, 2010.

[14] A. Cook and G. Tanner. European airline delay cost reference values. URL: http://www.eurocontrol.int/documents/european-airline-delay-cost-reference-values, 2011.

[15] N. Eggenberg, M. Salani, and M. Bierlaire. Constraint-specific recovery network for solving airline recovery problems. Computers \& Operations Research, 37(6):1014-1026, 2010.

[16] N. Jafari and S. H. Zegordi. Simultaneous recovery model for aircraft and passengers. Journal of the Franklin Institute, 348(7):1638-1655, 2011.

[17] A. I. Z. Jarrah, G. Yu, N. Krishnamurthy, and A. Rakshit. A decision support framework for airline flight cancellations and delays. Transportation Science, 27(3):266-280, 1993.

[18] L. Lettovsky. Airline operations recovery: an optimization approach. PhD thesis, Georgia Institute of Technology, 1997.

[19] L. Lettovsky, E. Johnson, and G. Nemhauser. Airline crew recovery. Transportation Science, $34(4): 337-348,2000$.

[20] S. J. Maher. Solving the integrated recovery problem using column-and-row generation. Transportation Science, 2014. Accepted.

[21] L. McCarty. Preemptive Rerouting of Airline Passengers under Uncertain Delays. PhD thesis, The University of Michigan, 2012.

[22] J. Petersen, G. Sölveling, E. Johnson, J. Clarke, and S. Shebalov. An optimization approach to airline integrated recovery. Transportation Science, 46(4):482-500, 2012.

[23] J. M. Rosenberger, E. L. Johnson, and G. L. Nemhauser. Rerouting aircraft for airline recovery. Transportation Science, 37(4):408-421, 2003.

[24] M. Stojković and F. Soumis. An optimization model for the simultaneous operational flight and pilot scheduling problem. Management Science, 47(9):1290-1305, 2001.

[25] M. Stojković and F. Soumis. The operational flight and multi-crew scheduling problem. Yugoslav Journal of Operations Research, 15(1):25-48, 2005.

[26] M. Stojković, F. Soumis, and J. Desrosiers. The operational airline crew scheduling problem. Transportation Science, 32(3):232-245, 1998. 
[27] B. G. Thengvall, J. F. Bard, and G. Yu. Balancing user preferences for aircraft schedule recovery during irregular operations. IIE Transactions, 32(3):181-193, 2000.

[28] G. Wei, G. Yu, and M. Song. Optimization model and algorithm for crew management during airline irregular operations. Journal of Combinatorial Optimization, 1(3):305-321, 1997. 